\title{
In-situ Tracking of Photochemical Ostwald Ripening Through Voltammetric Atom Counting
}

\begin{abstract}
Thomas R. Bartlett ${ }^{\dagger[a]}$, Stanislav V. Sokolov ${ }^{\dagger[a]}$, Blake J. Compton ${ }^{*[a]}$

Abstract: We report in-situ tracking of the atom count in individual particles during photochemical Ostwald ripening. The nano-impact technique, in conjunction with conventional UV-Vis and TEM analysis, is used to follow the photochemical formation of silver nano-prisms from spherical seed particles and a mechanism of Ostwald ripening is deduced.
\end{abstract}

Voltammetric impact studies of solution-phase nanoparticles (NPs) are important for fundamental, commercial and environmental applications ${ }^{[1]}$. Advances in this area have led to the successful characterisation of NPs through mediated processes ${ }^{[2]}$ and direct redox reactions ${ }^{[3]}$ with focus on the sizing of spherical geometries. In particular NPs colliding with an electrode held at a suitable potential are oxidised or reduced directly to provide quantitative atom count information. This technique has also been shown to provide sizing and aspect ratios of nano-rods ${ }^{[4]}$ and track NP reactions ${ }^{[5]}$. In the following, the nano-impact technique is applied to the study of NP synthesis to provide critical atom count information and new mechanistic insights.

The mechanistically informed synthesis of new NP geometries is potentially ground-breaking ${ }^{[6]}$. Nano-prisms of silver, synthesised by photochemical conversion of spherical seeds by Mirkin et al. ${ }^{[7]}$, are an example of a valuable NP geometry that gives rise to surprising properties ${ }^{[8]}$ and sensing applications ${ }^{[9]}$. Mechanistic studies for nano-prism formation have revealed the basic chemistry as shown in Scheme $1^{[10]}$. Reorganisation of $\mathrm{Ag}$ is rationalised by involvement of a redox cycle driven by citrate reduction of $\mathrm{Ag}^{+}$following $\mathrm{O}_{2}$ mediated oxidation of $\mathrm{Ag}^{0}$. This is assumed to lead to an Ostwald ripening process $^{[11]}$, whereby the consumption of smaller NPs provides material for growth of larger NPs. Study thus far has involved rapid conversion of seeds to prisms with either UV-Vis or ex-situ TEM used to probe key growth stages ${ }^{[12]}$. Statistical information and population changes during formation which could be gained by in-situ studies have proved elusive.

Herein we report a new method using nano-impacts to track NP synthesis and provide atom counting of individual NPs. The methodology outlined allows observation of key growth

[a] T. R. Bartlett, S. V. Sokolov, Dr. B. J. Plowman and Prof. R. G. Compton

Department of Physical and Theoretical Chemistry

Oxford University, South Parks Road

Oxford, OX13QZ (UK)

E-mail: richard.compton@chem.ox.ac.uk

[b] Dr. N. P. Young

Department of Materials

Oxford University, Parks Road

Oxford, OX13PH (UK)

$\dagger \quad$ Contributed equally to this paper

Supporting information for this article is given via a link at the end of the document.

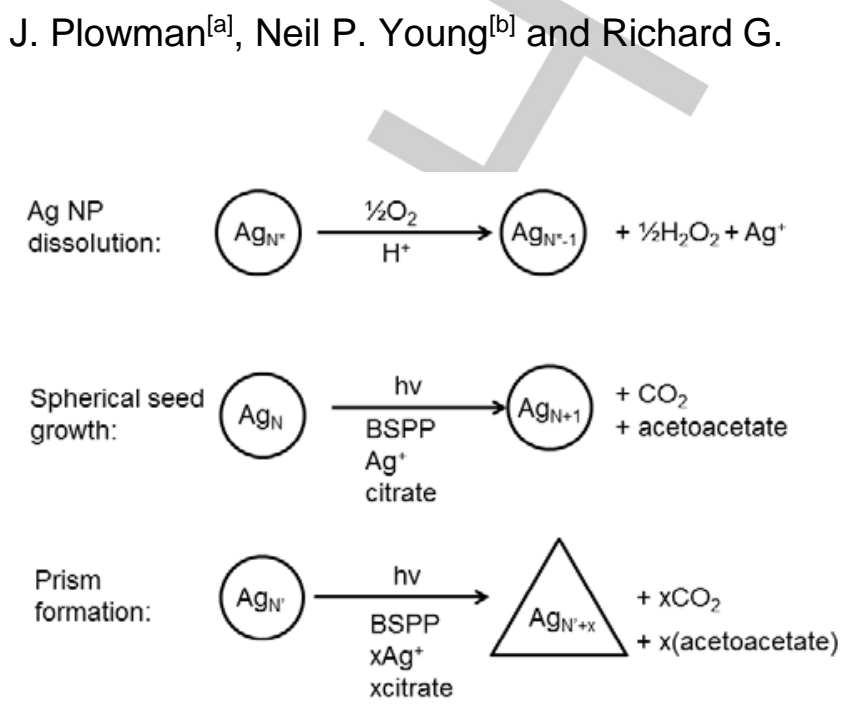

Scheme 1. Suggested mechanism for the photochemical transformation of spherical Ag seed NPs to nano-prisms ${ }^{[10]}$. BSPP $=$ bis (p-sulfonatophenyl) phenylphosphine. $\mathrm{O}=$ spherical NP. $\Delta=$ nano-prism

stages in terms of the number of atoms gained or lost by individual particles, unaffected by potential shape bias, and most importantly follows the growth of particles in-situ.

Silver nano-prisms capped with citrate and bis ( $p$ sulfonatophenyl) phenylphosphine (BSPP) were grown photochemically from spherical seed particles as reported by Mirkin et $a l^{[7 b]}$, except for using a much lower intensity light source (18 W, fluorescent tube, $138 \mathrm{~mm}$ distance) to markedly slow the process allowing better mechanistic resolution. Figure 1(a) shows an image of the NP stock suspension at daily intervals for 10 days. The colour of the suspension changes from yellow to blue, indicative of a change in NP geometry ${ }^{[7,10]}$. UV-Vis spectra for the NPs, as shown in Figure 1(b), were taken at daily intervals. A clear trend is apparent, with a concurrent decrease in the $\mathrm{P} 1$ and increase in P2 over the 10 day conversion period. $\mathrm{P} 1$ corresponds to the expected response for spherical seed NPs and P2 to the in-plane dipole resonance present for both nano-disk and nano-prism structures ${ }^{[7,10,13]}$. Figure 1(c) shows a plot of P1 and P2 peak absorbance variance with time. An approximately sigmoidal shape is observed and indicates the main change in absorbance occurs between days 2 and 7 . This period of rapid change in the P1:P2 peak absorbance ratio has previously been linked to the main growth period of the particles and indicates that under our conditions this occurs over a 5 day period. This confirms a slower rate of conversion than previously studied because of using a lower intensity light source ${ }^{[7,10]}$.

To observe geometrical transformations responsible for the UV-Vis changes detected, TEM images of the NPs were measured at intervals across the conversion period of 10 days as shown in Figure 2(a). Day 0 was shown to consist entirely of spherical seed particles of size $(5.9 \pm 2.5 \mathrm{~nm})$. By day 3 all NPs observed remained spherical, but with some larger particles now present. Approximately $15 \%$ of analysed particles displayed 
(a)

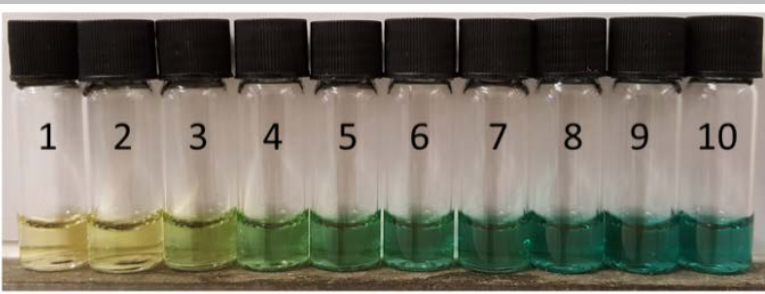

(b)
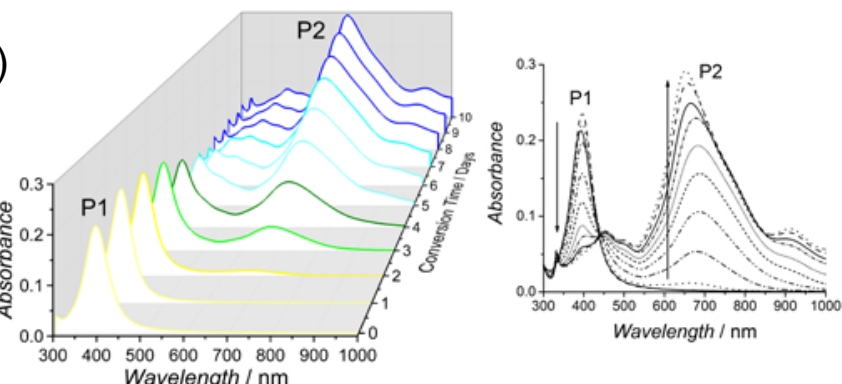

Wavelength / nm

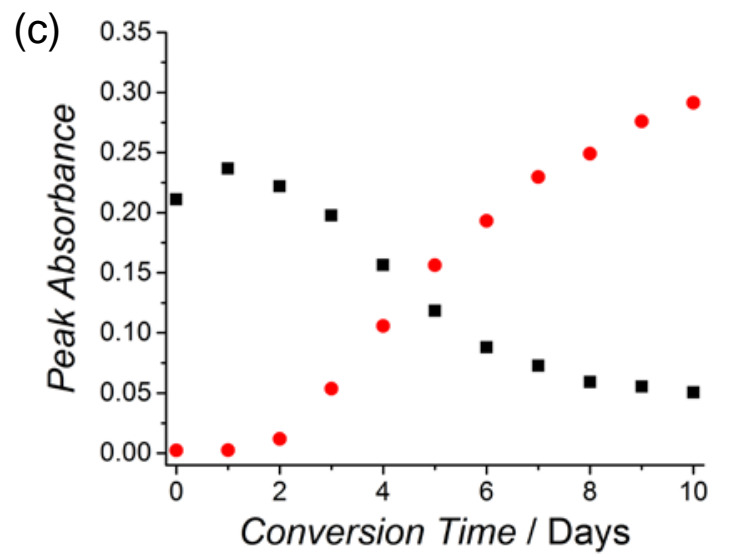

Figure 1. (a) Image of Ag NP suspensions showing colour change over the studied timescale of conversion. (b) UV-Vis spectra of the Ag NP suspension over the studied timescale of conversion. (c) Plot of peak absorbance vs. conversion time where black is the peak at $396 \mathrm{~nm}(\mathrm{P} 1)$ and red corresponds to P2.

planes of material extending from the core of the particle as evidenced by a lower contrast. These planes, highlighted in red in Figure 2(a), were only found on particles with diameter $>7 \mathrm{~nm}$ and to our knowledge have not been previously reported. They seem to show the beginnings of Ag deposition and growth on larger seed particles. Such planes are no longer visible by day 5 , where larger particles begin to form. Accurate analysis of particles on day 6 was hindered due to the irregularity of the NP geometries observed as exemplified in Figure 2(a). NPs of spherical geometry were still present and were all found to be below $10 \mathrm{~nm}$ in diameter. Days 8 and 10 showed the ultimate prismatic NPs, with some smaller spherical NP seeds still present.

To distinguish geometrical rearrangement and volumetric growth of particles over time, it is necessary to monitor the change in the number of atoms of Ag per NP. Figure 2 (b) shows the calculated distributions estimated from the TEM images across the conversion timescale. The distributions are split into "spherical" and "prismatic" contributions, expressed in terms of moles of $\mathrm{Ag}$ per NP, to allow direct comparison between different particle geometries. These were calculated using the following equations:
$M_{\mathrm{S}}=\frac{\frac{4}{3} \pi r^{3} p}{\mathrm{~A}_{\mathrm{r}}}$

$M_{\mathrm{p}}=\frac{\frac{1}{4} \sqrt{3} \mathrm{~d}^{2} \mathrm{~h} p}{\mathrm{~A}_{\mathrm{r}}}$

where $M_{s}$ and $M_{p}$ are the moles of Ag per NP for spherical and prismatic NPs respectively, $r$ is the radius of the spherical NP, $p$ is the density of $\mathrm{Ag}\left(10.49 \mathrm{~g} \mathrm{~cm}^{-3}\right)^{[14]}, A_{r}$ is the atomic mass of $\mathrm{Ag}$, $d$ is the edge length of the prisms and $h$ is the thickness of the prism. This thickness was measured from TEM images of stacked prisms and was consistent for prismatic particles across the days where they appear at $(6.7 \pm 0.8 \mathrm{~nm})$. This value was used for calculations of moles Ag per NP for all prisms. An example TEM image of the stacked prisms can be seen in figure 2(a) day 8(ii) and the distribution of the prism thickness in SI 3(v). A broadening of the distribution and $a$ fall in the relative population of smaller particles is observed during the conversion timescale. The beginnings of ripening seem to be visible on day 3 , with a slight tail in the distribution towards higher atom count. A fall in the proportion of smaller particles and slight broadening of the distribution from day 3 to 5 confirms the observation of the formation of larger particles. Note that the UV-Vis data shows a rapidly increasing signal at this period indicating a change in NP geometry. This is in contrast with TEM where only spherical particles are observed. Nano-disks are known to have similar UV-Vis absorbance to nano-prisms ${ }^{[13]}$ and are difficult to differentiate from spheres without electron emission loss spectroscopy (EELS) due to their similar 2-D shape. Therefore it can be inferred from the UV-Vis spectra that nano-disks are formed prior to prism formation. From day 5 to 8 the most significant change in distribution occurs, along with the appearance of prisms. This shows that the formation of the prismatic geometry occurs concurrently with an increase in atom count of the particle. The formation of prisms results in a decrease in the proportion of small spherical particles. By day 10 the distribution primarily consists of triangular nano-prisms. The consumption of small particles and growth of larger particles is consistent with an Ostwald ripening process. However due to the complex geometries of particles, especially at day 6, accurate sizing and volumetric information was difficult to obtain. Therefore, to complement the TEM results an in-situ study of the conversion was conducted concurrently using nano-impact analysis.

Nano-impact voltammetry allows for non-shape bias analysis of NPs based only on the number of atoms in the NP ${ }^{[15]}$ As the technique is not influenced by the assumption of idealised geometries as for the TEM analysis, it may provide a more accurate description of particle growth for individual NPs. In addition, the speed and cost of use allows for samples to be measured at more frequent intervals than TEM imaging. For accurate distributions of the particles to be obtained it was necessary to minimise aggregation or agglomeration effects in electrolyte. Studies have shown prisms to be especially susceptible to inorganic anion composition, undergoing geometric reconfiguration when exposed to chloride, yet retaining their prismatic nature in nitrate ${ }^{[16]}$. The long term stability of nano-prisms in nitrate however has not been reported.

Particle stability in the chosen electrolyte, $40 \mathrm{mM} \mathrm{KNO}_{3}$, was ensured by UV-Vis analysis of the particles at $5 \mathrm{~min}$ intervals for 2 hours at 24 hour intervals, the results of which can be viewed in the SI. The spherical and prismatic peaks were 
(a)
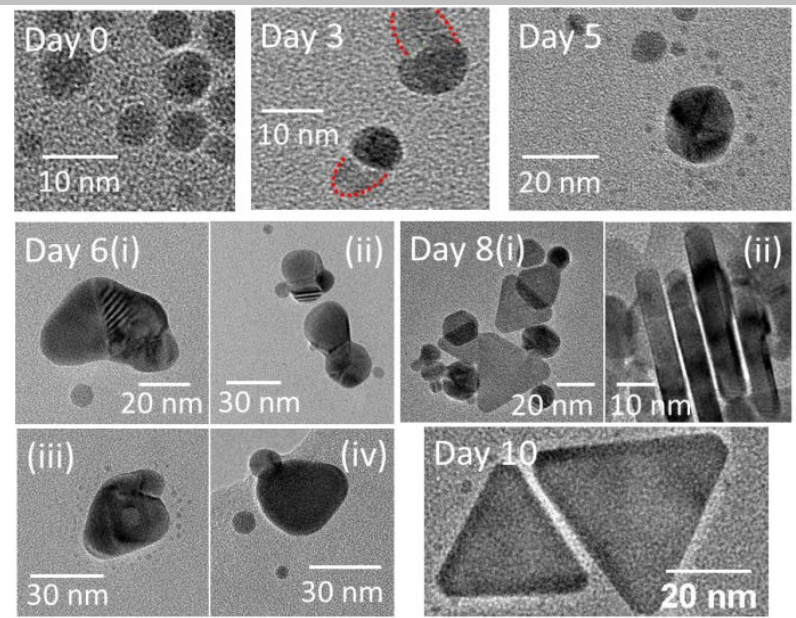

(b)

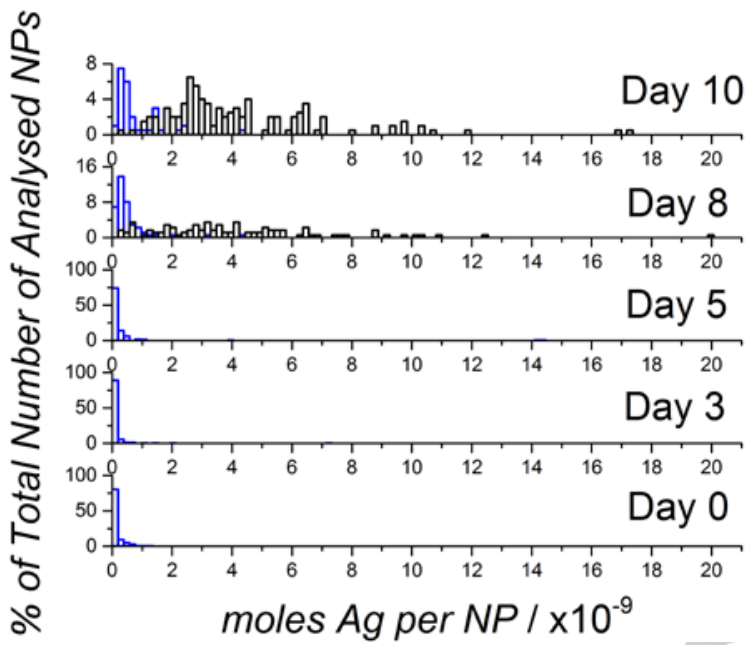

Figure 2 (a) Example TEM images. (b) TEM distributions for the NPs over the timescale of conversion. Blue fractions are spherical particles and black fractions are prisms. The number of particles analysed for the days $0,3,5,8$ and 10 were $160,157,158,167$ and 200 respectively.

found to be stable under these conditions, with little broadening or reduction in height of the peaks observed due to aggregation ${ }^{[17]}$. Nano-impact experiments were conducted by diluting samples of the NP stock taken at daily intervals by 20 times in $40 \mathrm{mM} \mathrm{KNO}_{3}$. The suspension was not degassed as this was found to induce aggregation or agglomeration of the prisms, noted by a loss of colour of the solution. Current-time transients were recorded at an oxidising potential of $+0.40 \mathrm{~V}$ vs MSE. Current spikes were integrated using signal counter software ${ }^{[18]}$ and equation 3 used to calculate the moles of $\mathrm{Ag}$ per particle, $M$ :

$M=\frac{Q}{n F}$

where $Q$ is the charge of the integrated impact spike, $n$ is the number of electrons transferred to each $\mathrm{Ag}$ atom $(n=1)$ and $F$ is the Faraday constant. An example of the current-time transient responses is in the SI.

Figure 3 shows the resultant distributions from nanoimpacts conducted for each day during conversion Across the 10 day conversion period a broadening of the particle distribution is observed. This occurs concurrently with a reduction in the number of small particles detected. The most
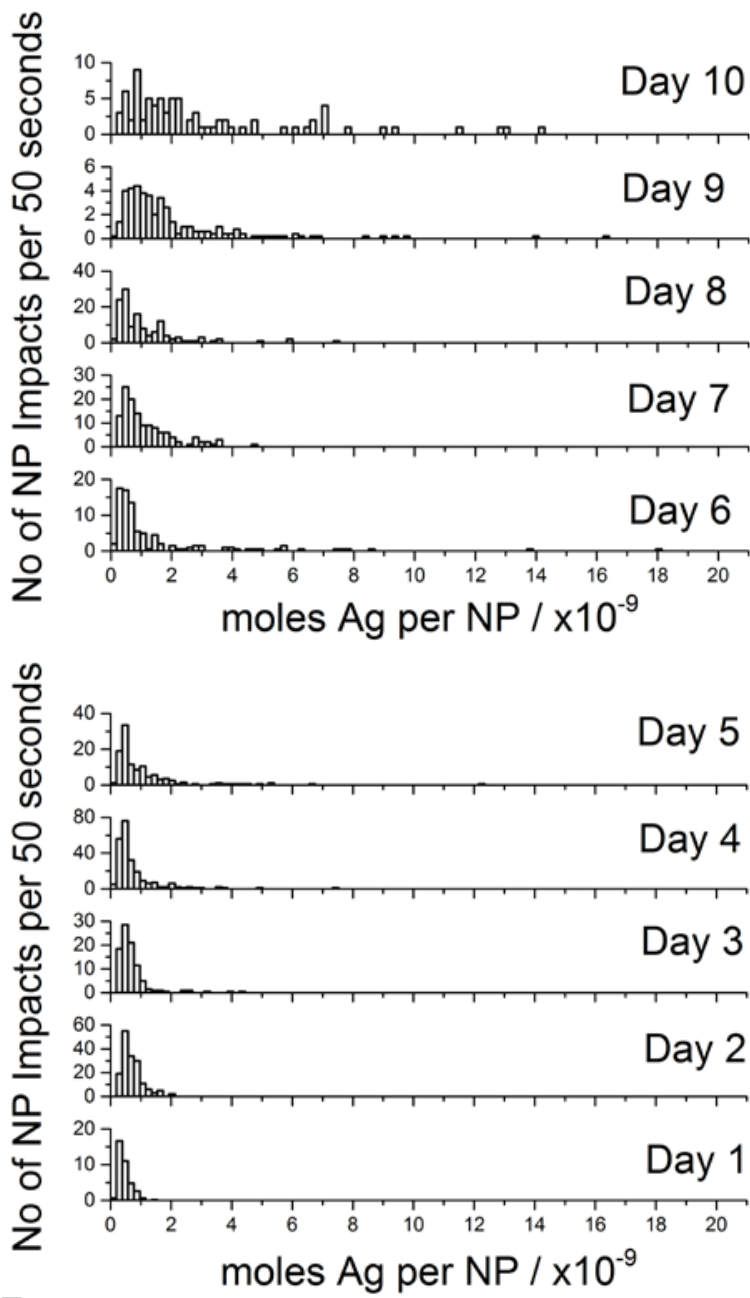

Figure 3. Distributions of impacted NPs at daily intervals during conversion gained by nano-impact voltammetry of the NP suspension 20 times diluted in $40 \mathrm{mM} \mathrm{KNO}_{3}$ at $+0.40 \mathrm{~V}$ vs MSE.

significant shift in distribution occurs from day 8 to 10.This is consistent with the TEM analysis and shows the use of nanoimpacts for tracking NP growth. TEM and nano-impact distributions on the same days show good agreement with the small deviations arguably attributed to the perfect geometric assumptions made in the TEM analysis. As nano-impacts are not affected by particle shape and purely probe the atom count per NP, all stages could be analysed including day 6 where accurate sizing by TEM could not be obtained. The resultant nano-impact distribution indicates that the irregular shaped particles observed on day 6 (see Figure 2 (a)) do contain particles of increased atom count. However the distribution is still strongly reminiscent of the starting particles with most particles possessing less than $1 \times 10^{-9}$ moles $\mathrm{Ag}$ per NP. Significant distribution changes only occur from day 8 to 10 , which indicates that the main growth period of the particles occurs much later on through the conversion than the geometric changes indicated by UV-Vis analysis. This highlights the surface sensitive nature of UV-Vis spectroscopy and in this case its limitations in tracking growth in these types of processes.

The slow timescale of conversion and concurrent TEM and nano-impact analysis allows us to propose the mechanism for Ostwald growth of nano-prisms as shown in Scheme 2. Here, dissolution of small Ag seeds and growth on larger NPs occurs, 
with the formation of 2D planes on larger NP seeds. Next, ripening of these particles forms nano-disk intermediates which give rise to the initial changes in UV-Vis absorbance. These nano-disk-like structures then continue to grow into the prismatic structures observed at day 8 and 10. The formation of the prismatic geometry occurs with an inherent increase in atom number.

In summary, the nano-impacts technique has been shown to track NP growth by Ostwald ripening, providing in-situ atom counting information of individual NPs. This is found to correlate with ex-situ TEM imaging and newly indicates the role of a nanodisk geometry in the conversion of spherical NPs to nano-prisms. With accurate volumetric information gained from nano-impact analysis, it can be inferred that geometric changes to the particles occurs prior to growth. As nano-impacts are not affected by the shape of the particles it provides a complemetary method for following growth in such systems where UV-Vis spectroscopy is relatively insensitive to some geometric changes This method of following particle growth by nano-impact voltammetry is broadly applicable and is a low cost and fast method for following NP synthesis whilst also providing mechanistic insights, statistical information and population distributions.
677; c) A. Albanese, P. S. Tang, W. C. Chan, Annu. Rev. Biomed. Eng. 2012, 14, 1-16.

a) R. Jin, Y. Cao, C. A. Mirkin, K. L. Kelly, G. C. Schatz, J. G. Zheng, Science 2001, 294, 1901-1903; b) R. Jin, Y. C. Cao, E. Hao, G. S. Metraux, G. C. Schatz, C. A. Mirkin, Nature 2003 $425,487-490$.

a) A. K. Singh, D. Senapati, A. Neely, G. Kolawole, C. Hawker, P. C. Ray, Chem. Phys. Lett. 2009, 481, 94-98; b) J. Nelayah, M. Kociak, O. Stephan, N. Geuquet, L. Henrard, F. J. Garcia de Abajo, I. Pastoriza-Santos, L. M. Liz-Marzan, C. Colliex Nano Lett. 2010, 10, 902-907; c) N. L. Gruenke, M. F. Cardinal, M. O. McAnally, R. R. Frontiera, G. C. Schatz, R. P. Van Duyne, Chem. Soc. Rev. 2016, in press.

M. M. Shahjamali, M. Salvador, M. Bosman, D. S. Ginger, C Xue, J. Phys. Chem. C. 2014, 118, 12459-12468.

C. Xue, G. S. Metraux, J. E. Millstone, C. A. Mirkin, J. Am. Chem. Soc. 2008, 130, 8337-8344.

W. Z. Ostwald, Phys. Chem. 1901, 37, 385. Ed. Engl. 2007, 46, 8436-8439.

P. Sarkar, D. K. Bhui, H. Bar, G. P. Sahoo, S. Samanta, S. Pyne, A. Misra, Nanoscale Res. Lett. 2010, 5, 1611-1618.

Handbook of Chemistry and Physics, 55 ed., CRC Press, 1974.

S. S. Sokolov, C. Batchelor-McAuley, K. Tschulik, S. Fletcher, R. G. Compton, Chem. - Eur. J. 2015, 21, 10741-10746.

X. C. Jiang, A. B. Yu, Langmuir 2008, 24, 4300-4309.

S. Botasini, E. Méndez, J. Nanopart. Res. 2013, 15, 1526. W. Cheng, R. G. Compton, Angew. Chem., Int. Ed. Engl. 2014, $53,13928-13930$.

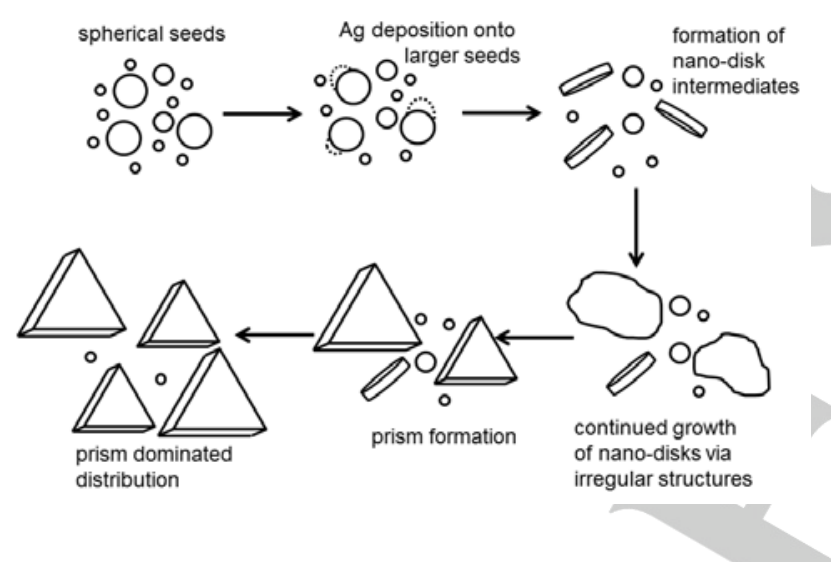

Scheme 2. Schematic mechanism proposed for the photochemical Ostwald ripening of spherical Ag NPs to nano-prisms via nano-disk intermediates.

\section{Acknowledgements}

The authors of this work acknowledge funding from the European Research Council (ERC) under the European Union's Seventh Framework Programme (FP/2007-2013)/ERC Grant Agreement no. [320403] (TRB, SVS, RGC) and the Marie Curie International Incoming Fellowship, project number [630069] (BJP).

Keywords: nano-impacts $•$ nanoparticle $\bullet$ nano-prism $•$ Ostwald ripening $\bullet$ silver

\footnotetext{
[1] M. Pumera, ACS Nano 2014, 8, 7555-7558.

X. Xiao, A. J. Bard, J. Am. Chem. Soc. 2007, 129, 9610-9612.

N. V. Rees, Electrochem. Commun. 2014, 43, 83-86.

B. J. Plowman, N. P. Young, C. Batchelor-McAuley, R. G. Compton, Angew. Chem., Int. Ed. Engl. 2016, in press.

5] T. R. Bartlett, R. G. Compton, Russ. J. Electrochem. 2016, in press.

[6] a) J. J. Mock, M. Barbic, D. R. Smith, D. A. Schultz, S. Schultz, J. Chem. Phys. 2002, 116, 6755; b) K. L. Kelly, E. Coronado,
} L. L. Zhao, G. C. Schatz, J. Phys. Chem. B. 2003, 107, 668- 


\section{COMMUNICATION}

Counting atoms! Prismatic transformation of spherical silver nanoparticles by light is tracked in-situ allowing the number of atoms per individual particle to be measured throughout the conversion.

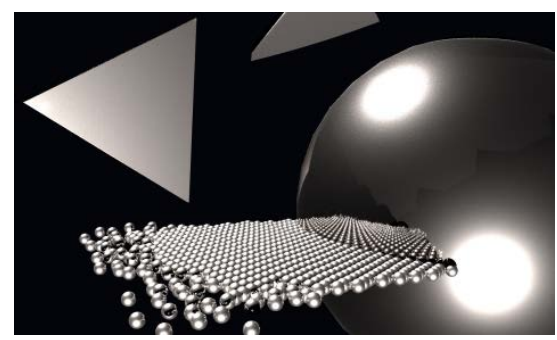

Author(s), Corresponding Author(s)*

Page No. - Page No.

Title 